\title{
Recensement et examen critique des méthodes d'observation de la cavitation par voie acoustique
}

\author{
PAR M. RATA, \\ INGÉNIEUR AU DÉPARTEMENT 《ESSAIS》 \\ DU CENTRE DE REGHERches ET D'ESSAIS DE CHATOU (E.D.F.)
}

\begin{abstract}
Ce texte a été mis au point dans le cadre de la Commission de la Cavitation de la Société Hydrotechnique de France, par le Groupe de travait $n^{\circ} 4$, dont la composition élait la suivunte : MM. RATA (C.R.E.C.E.d.F.), BINDEL (Bassin des Carènes), OSTERWALDER (Société Escher Wyss) et PEARSALL (National Engineering Laboratory).

On trouvera dans le corps de l'article :

- les résultats d'une enquête portant sur les moyens mis en ouvre dans les divers laboratoires pon* déterminer l'apparition et le développement de la cavitation par voie acoustique;

- l'étude critique de ces différents moyens et de leur efficacité.
\end{abstract}

\section{I. - INTRODUCTION}

La détection et l'observation de la cavitation par voie acoustique semble jusqu'à présent assez peu utilisée, sauf peut-être dans certains cas spéciaux.

Nous avons questionné divers laboratoires de recherche industrielle, de manière à présenter une vue aussi complète que possible de ce qui se fait actuellement en ce domaine, et il nous est apparu très nettement que les laboratoires axés sur la recherche d'une limite de cavitation dans une machine hydraulique n'utilisaient pratiquement pas ces méthodes.

La cavitation, par les ondes de choc produites lors de l'implosion des bulles de vapeur, semble a priori pouvoir aisément être étudiée par voie acoustique.

Cependant, la plupart des expérimentateurs de stations d'essais ne font qu'occasionnellement des mesures de bruit : beaucoup de paramètres, autres que la cavitation elle-même, entrent en jeu, il n'a pas encore été possible de séparer leurs influences.

Des études plus approfondics sont nécessaires pour aboutir à une meilleure compréhension de ce phénomène.

\section{II. - LES CHAMPS D'ETUDE DE LA CAVITATION}

Les machines étudiées dans les divers laboratoires comprennent des turbines, pompes, hélices, -vannes, venturis, dont les dimensions varient de 0,20 à $1,00 \mathrm{~m}$. D'autre part, ces laboraloires effectuent des essais sur modèles.

A part la Station d'écoute de la Marine du Brusc et le Laboratoire de Physiologgie appliquée de Jouy-en-Josas, aucune des personnes contactées n'effectue de manic̀re systématique 
des mesures de bruit. La plupart ne font de mesures de ce type que de façon occasionnelle, souvent pour essayer de mettre an point une méthode.

\section{III. - RENSEIGNEMENTS QU'APPORTE L'ANALYSE DU BRUIT DANS L'ETUDE DE LA CAVITATION}

L'analyse du bruit apporte des précisions sur le début de cavitation, est-il généralement admis, et des indications sur le développement de celleci. Le niveau de bruit présente en effet un accroissement assez marqué dès que les implosions de cavités débutent, si la transmission acoustique du milieu ne change pas durant les mesures, par exemple par variation de sa teneur en air, ou formation de dispersions gazeuses.

L'on peut objecter à ce genre de mesure, effectuée sur modèle, qu'elle ne peut permettre de distinguer entre la cavitation qui peut se produire sur un défaut de surface, et la cavitation due au profil étudié.

Il est évident que l'observation visuelle fournit dans ce cas la possibilité d'interpréter les bulles de cavitation que l'on peut voir, et d'éliminer ce qui est dô à une aspérité.

Cependant, les deux méthodes se complètent, car, le plus souvent, l'augmentation du bruit a lieu un peu avant les premières bulles visibles, ce qui permet à l'observateur d'apporter plus d'attention à son examen dès cette élévation du niveau.

De plus, dans le cas de la machine industrielle, l'étude du bruit est la seule qui permette de caractériser un état de cavitation par rapport au régime non cavitant.

L'augmentation de la cavitation provoque une élévation concomitante du niveau de bruit, et ce dernier peut donc permettre, dans les conditions de transmission constantes, d'évaluer le degré de cavitation.

Un des points remarquables, que nous avons vérifié à plusieurs reprises sur la plate-forme d'essais de Chatou, est la reproductibilité de ces mesures de bruit, toutes choses égales d'ailleurs. A plusieurs mois d'intervalle, on retrouve la même courbe de bruit, à $1 \mathrm{~dB}$ près.

Par contre, au-delà d'un certain niveau de cavitation, le bruit diminue, vraisemblablement à cause de l'absorption acoustique des bulles dégagées dans le fluide. Cette particularité empêche d'établir une correspondance degré de cavitationbruit au-delà d'une certaine limite.

Les essais sur machines (grandeur nature), cependant, sont beaucoup moins aisés; le phénomène de cavitation est souvent masqué dans un bruit de fond d'origines diverses, et dont il est assez difficile de séparer les effets de ceux de la cavitation.

Au point de vue de la localisation sur les organes, l'état actuel des méthodes acoustiques d'étude ne permet pas d'avoir de renseignements. Il en est de même pour l'érosion que l'on peut attendre de la cavitation; dans certains cas, des points de fonctionnement très bruyants ne produisent guère d'érosion, alors que d'autres d'un niveau acoustique bien plus faible produisent des érosions importantes. Cela peut être lié au fait que la courbe de bruit diminue au-delà d'une certaine limite.

\section{IV. - LES METHODES ACOUSTIQUES D'ÉTUDE DE LA CAVITATION}

L'étude du bruit de cavitation peut se faire de trois manières: en mesurant le niveau sonore global, en analysant le spectre de fréquence du bruit, et enfin en analysant son spectre d'amplitude. La mesure n'est significative que si la teneur en air et la constitution physicue du milieu ne varient pas en cours de mesures.

\section{La méthode du bruit global.}

C'est la plus répandue et la plus facile à mettre en œuvre. Un capteur est placé en un point de la machine, au voisinage de la source de bruit, et l'on effectue la mesure du niveau de sortie de ce capteur, en grandeur moyenne ou efficace, après une amplification éventuelle.

La bande passante du capteur détermine pratiquement la gamme de fréquences du signal mesuré. Celle-ci s'étend, selon les utilisateurs, de $100 \mathrm{~Hz}$ à quelques centaines de $\mathrm{kHz}$. Il est quelquefois avantageux de procéder à une coupure des basses fréquences, dans lesquelles on trouve toutes les vibrations mécaniques de la machine.

Il est avantageux, dans ce genre de mesure, de prendre la vraie valeur efficace du signal, au moyen d'un thermocouple, par exemple, plutôt que la valeur moyenne, car le signal est souvent de forme impulsionnelle, et son facteur de forme est variable.

Le capteur utilisé peut être étalonné en pressions, par exemple en volts par baryes, ce qui permet de faire des mesures absolues; le plus souvent, cependant, ce sont des mesures relatives qui sont intéressantes pour comparer le niveau de bruit à un certain état de cavitation avec un niveau de référence dans un état non cavitant. 


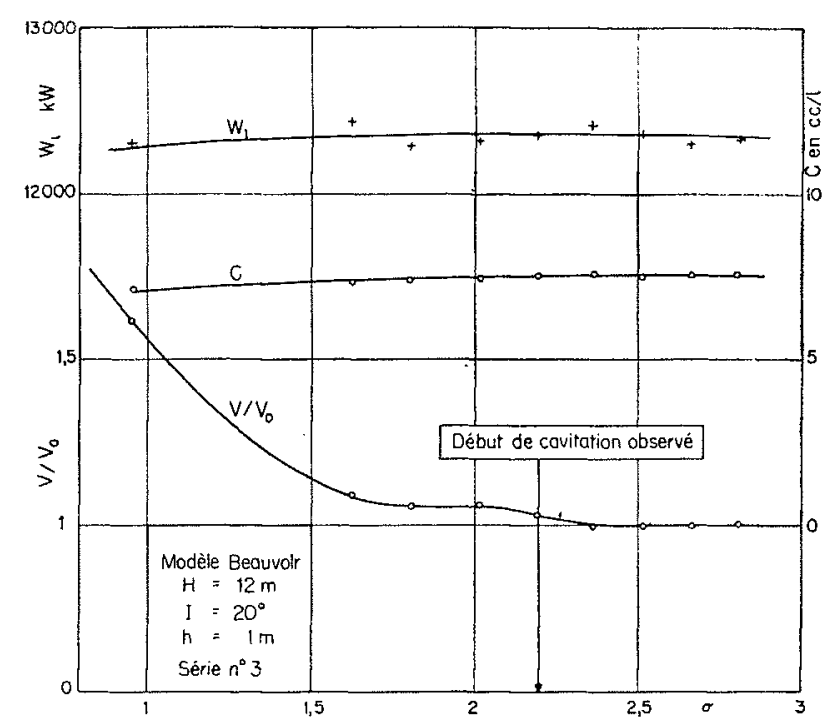

Fig. 1

On peut aussi employer des accéléromètres placés sur la machine, ces derniẹrs ayant l'inconvénient d'être soumis à toutes les vibrations de la machine, en plus de celles dues au phénomène étudié.

L'exploitation de la mesure se fait en tracant la courbe de variation du niveau de bruit en fonction du paramètre de cavitation, soit en valeurs absolues, ou, mieux encore, en valeurs relatives, ce qui élimine l'influence de la transmission des parois.

Sur les figures 1 et 2 , on peut voir une telle courbe de bruit en fonction du $\sigma$, relevée par M. Cormault sur la Station d'Essais de Chatou : on peut constater que le niveau de bruit augmente déjà un peu avant l'apparition des premières bulles visibles.

Signalons que l'on peut corriger dans une certaine mesure les variations d'absorption $\mathrm{du}$ milieu (rapport de M. Cormault à la Société Hydrotechnique de France, VITes Journées de l'Hydraulique, Paris).

\section{La méthode de l'analyse de fréquence.}

Pour essayer d'augmenter l'information fournie par le bruit de cavitation, on peut en effectuer l'analyse du spectre de fréquences.

Ceci peut être fait de deux façons différentes : en tiers d'octave, ou en continu. Dans l'analyse en tiers d'octave, on fait passer le signal dûment amplifié à travers une batterie de filtres passebande, dont la largeur de bande est proportionnelle à la fréquence, et dont on utilise trois unités par octave. La gamme de fréquences analysée va de $16 \mathrm{~Hz}$ à $50 \mathrm{kHz}$.

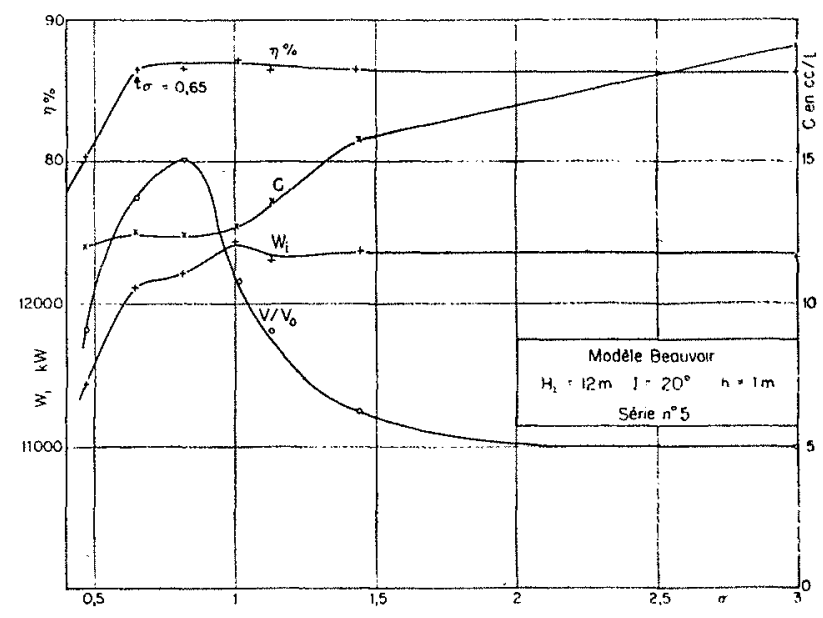

F1G. 2

A la sortie de ces filtres, le niveau du signal est mesurć, en grandeur efficace sénćralement.

Dans l'analyse en continu, on fait passer le signal à travers un filtre à fréquence variable, mais de largeur de bande constante. La bande couverte va de $100 \mathrm{~Hz}$ à $100 \mathrm{kHz}$ en général.

Pour chaque valeur du paramètre de cavitation, on dresse ainsi une courbe, constituant le spectre de fréquences du signal.

L'exploitation de la méthode se fait en traçant, à fréquence donnéc, la courbe de variation du niveau de signal en fonction du paramètre de cavitation.

On trouve généralement que ces courbes sont assez voisines les unes des autres, et présentent une augmentation comparable à celle du bruit global.

Le spectre de fréquence, toutefois, se déforme avec le degré de cavitation. Les résonances de la machine sont toujours indiquées par le spectre de fréquence.

On peut voir (fig. 3 et 4 ) une famille de spectres de fréquences en tiers d'octave, et les courbes correspondant à diverses fréquences en fonction du paramètre de cavitation.

\section{La méthode de l'analyse d'amplitude.}

On peut, au contraire, découper le signal incident en tranches d'amplitude, et compler le nombre de fois que les crêtes du signal se trouvent dans l'une de ces bandes, pendant un temps connu.

La figure 5 montre un exemple sommaire de comptage en cinq tranches d'amplitude d'un signal théorique.

Chaque valeur du paramètre de cavitation fournit aussi un spectre d'amplitude, similaire 
BRUIT DE CAVITATION

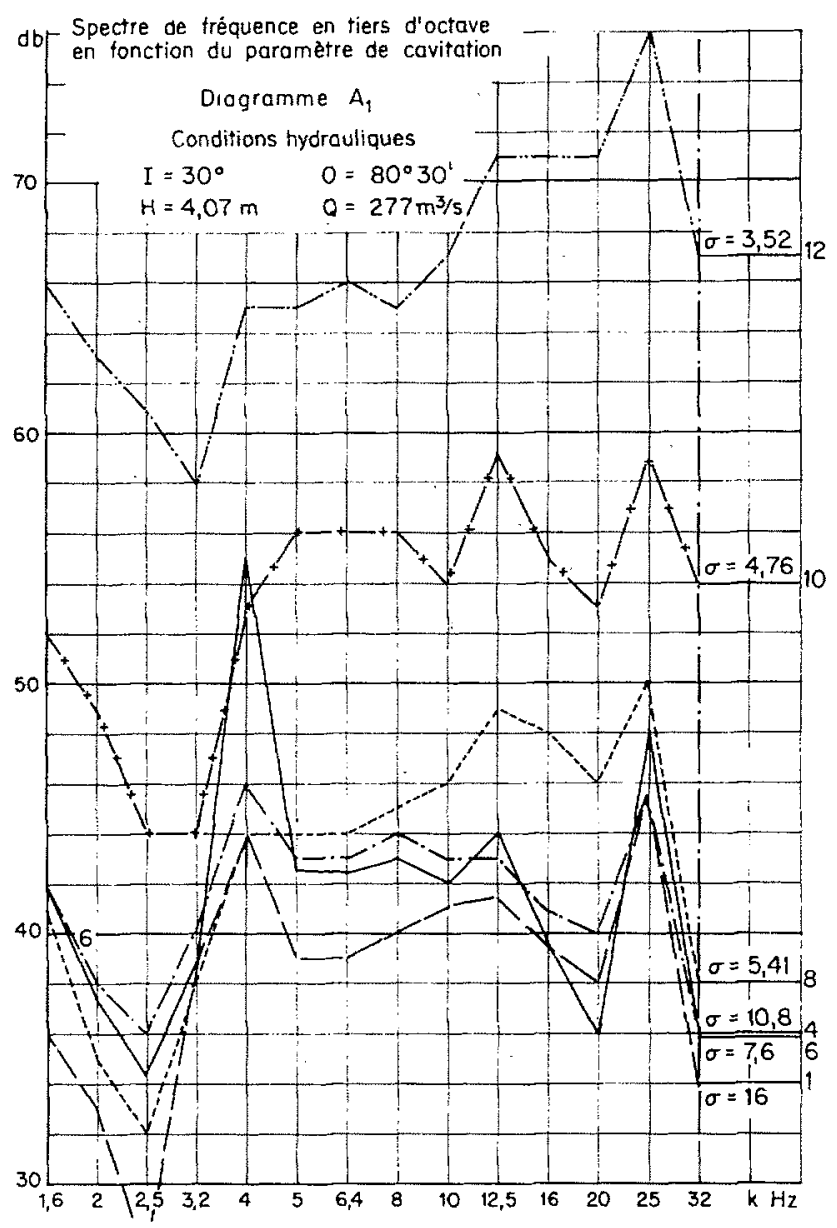

Fig. 3

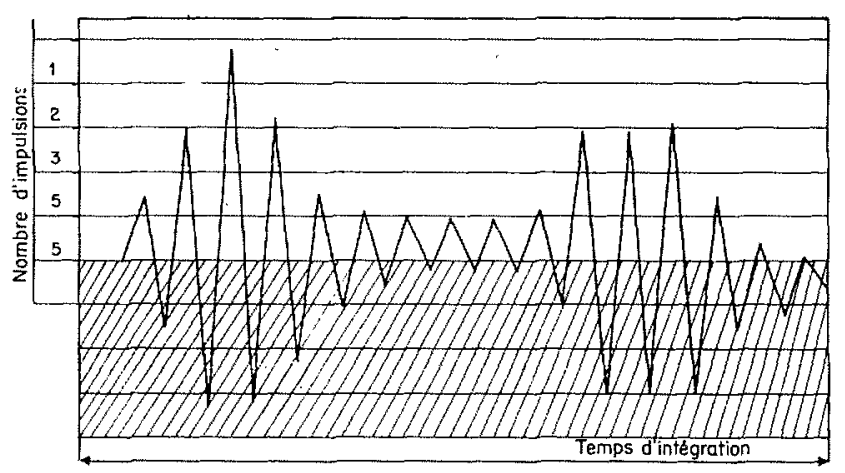

FIG. 5

au spectre théorique de la figure 6 , correspondant à l'exemple précédent.

L'exploitation de la méthode est analogue à
BRUIT DE CAVITATION

Courbes $\frac{V}{V_{0}}=f\left(\frac{\sigma}{\sigma_{0}}\right)$ pour $\sigma_{0}=16$ et diverses bondes de fréquences

Diogramme $\mathrm{A}_{2}$

Conditions hydrauliques

$I=30^{\circ} \quad O=80^{\circ} \cdot 30^{\prime}$.

$H=4,07 \mathrm{~m} \quad \mathrm{Q}=277 \mathrm{~m}^{3} / \mathrm{s}$

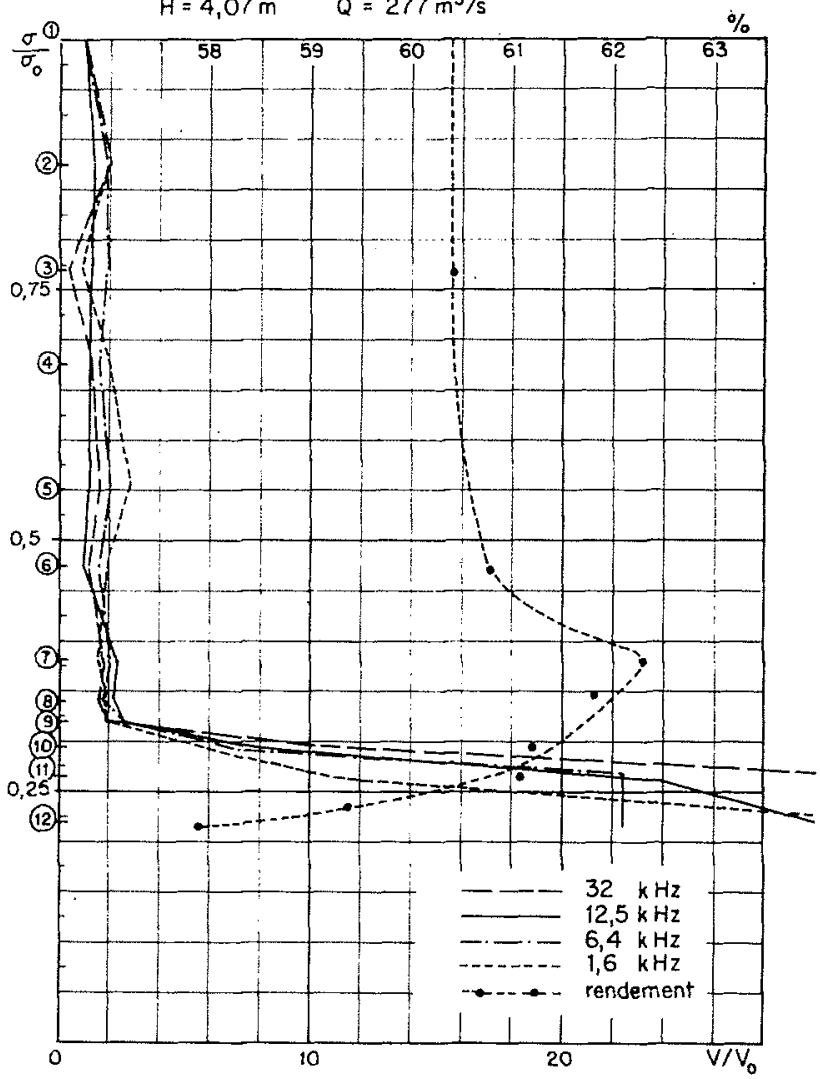

FIG. 4

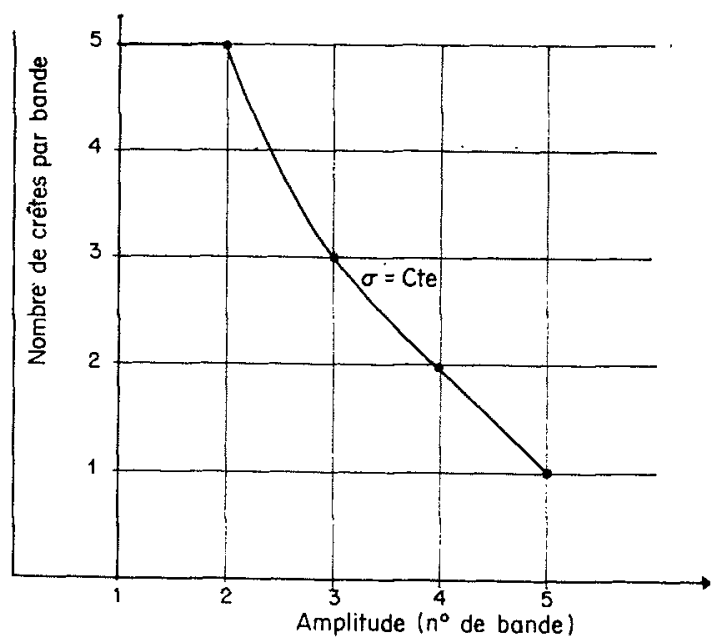

FIG. 6

celle de l'analyse de fréquence : on trace les courbes du nombre de crêtes par bande en fonction $d u \quad \sigma$, pour une bande donnée. 


\section{V. - TECHNIQUE ET APPAREILLAGE UTILISES}

La partie la plus importante de la chaîne de mesure du bruit est sans contredit le capteur. On utilise généralement des capteur's piézoélectriques, en quartz, en sel de Seignette ou en titanate de baryum, quoique certains utilisateurs aient adopté des capteurs électromagnétiques.

La réponse en fréquence s'étend de quelques dizaines de $\mathrm{Hz}$ à quelques dizaines de $\mathrm{kHz}$, éventuellement à des fréquences plus élevées, de l'ordre de quelques centaines de $\mathrm{kHz}$.

On étalonne ces capteurs en pressions, comme par exemple le fait la Marine nationale au Brusc,

Quelquefois, cependant, on utilise des accéléromètres du même type, que l'on peut étalonner éventuellement sur table vibrante.

L'emplacement du capteur est aussi un des points importants de la mesure : l'élément sensible doit se trouver le plus près possible de la source de bruit; si l'on peut le faire, il ne faut pas hésiter à le mettre en paroi, en conlact direct avec l'écoulement, et isolé mécaniquement de la machine par des joints souples. Ceci diminue considérablement l'influence des parois sur la transmission, quoique des essais comparatifs entre deux hydrophones dont l'un était placé en paroi et l'autre sur la paroi, à l'extérieur, avec une eapacité de couplage constituée par un bain d'eau ou d'huile, ont montré que les indications de ces appareils étaient, à très peu près, équivalentes.

Les amplificateurs utilisés sont du type classique, avec cependant la nécessité d'avoir de fortes impédances d'entrée sur les capteurs piézoélectriques.

Les appareils de mesure sont de préférence du type à thermocouple, de manière à donner directement la grandeur efficace du signal. On utilise des constantes de temps assez grandes, de quelques secondes à $2 \mathrm{mn}$, pour éliminer les fluctuations.

\section{VI. - COMPARAISON ENTRE LES DIVERSES MÉTHODES}

Les méthodes d'étude de la cavitation que nous venons de passer en revue ont des aspects complémentaires. Le seuil d'apparition de la cavitation est généralement assez bien défini par la courbe de bruit global qui est facile à relever, et ne demande pas un appareillage compliqué.

La concordance des observations visuelles avec le bruit global est assez satisfaisante, quant au seuil d'apparition.
Jusqu'à présent, les autres méthodes n'ont guc̀re apporté de précisions supplémentaires quant au seuil. Il semble toutefois que l'analyse d'amplitude puisse fournir une variation plus brutale de la courbe du nombre d'impulsions que les autres méthodes au moment du seuil.

Mais c'est surtout dans l'évolution de la cavitation que les analyses de frécfuence et d'amplitude sont intéressantes : divers essais ont montré que les spectres se déforment an cours de l'évolution de la cavitation.

D'après le Laboratoíre du National Engineering à East Kilbride, on constate un accroisscment du bruit en haute fréquence $(10$ à $30 \mathrm{kHz})$ au voisinage du seuil de cavitation, cependant qu'en régime de cavitation développée, ce sont surtout les basses fréquences (quelques centaines de $\mathrm{Hz}$ ) qui augmentent.

La forme de la courbe de bruit varie en fonction du point de fonctionnement, semble-t-il, à cause vraisemblablement de la localisation différente des poches de cavitation sur les organes.

Des essais systémaliques sont eependant nécessaires pour préciser les relations éventuclles entre les spectres de bruit, l'évolulion de la cavitation et la localisation de celle-ci sur les organes.

\section{VII. - CONCLUSIONS}

Le peu d'intérêt porté aux méthodes de détection acoustique de la cavitation dans certains laboratoires est dû à la complexilé du phénomène de cavitation lui-même. Le bruit ne peut en effet supplanter l'observation visuelle pour la localisation des bulles de cavitation. Il est d'autre part impossible, dans l'état actuel de la technique, de donner des indications, au vu d'une courbe de bruit, sur le degré de cavilation de la machine, sur la localisation de celte cavitation.

Il est cependant indéniable que l'ctude du bruit peut se révéler être un auxiliaire précieux pour l'observateur au voisinage du seuil d'apparition. Il reste à espérer que nombre d'utilisateurs occasionnels de ces méthodes auront à cour d'essayer d'approfondir nos connaissances du problème, en faisant des relevés plus systématiques du bruil de cavitation. L'expérience alors mise en commun permettra de voir les possibilités réelles de la méthode.

\section{BIBLIOGRAPHIE}

1. P. Copmaurt. - Contribution à l'étude de l'influence des teneurs en faz de l'eau sur la cavitation dans les turbomachines hydrauliques (these Toulouse, juin 1962). 
2. P. Conmault. - Incidence des teneurs en gaz de l'eau sur la mesure du bruit comme critère d'apparition de la cavitation. VII Journées de l'Hydraulique, Question IV.

3. J. M. Rata. - Bruit de cavitation. Symposizm de Nice, 1960.

4. J. M. Rata. - Détermination aconstique du seuil de cavitation. VII Journées de l'Hydraulique, Question IV.

5. F. G. Blake. - Apparatus and Techniques for a study of cavitation. Jr. Horward University, Acoustics Research Laboratory, T. $\mathrm{n}^{0}$ 11, June 28, 1949.
6. WV. W. WuLmonTr. - Smell barium titanate transduces for aerodynamic or acoustic pressure measurements. The Review of Scientific Instruments. Vol. $29, n^{\circ} 3$, pages 218-222.

7. H. M. Fitzpatrick et M. Strasberg. - Sources and characteristics of Hydrodynamic noise. Proc. ONR.-MRC. Symposium on Naval Hydrodynamics, Washington DC.

8. Bishop and Salmich. - Noise, vibrations and measurements problems resulting from fluid flow disturbance. Trans. of the ASME, Vol. 79, $\mathrm{n}^{\circ} 5$, juillet 1953.

9. BoLM. - Sound spectre and acoustic pressure distribution for cavitation. Acustica, 7-4, 1957.

\title{
DIS CUSSION
}

Président : M. BERGERoN

M. le Président rappelle que ce rapport, présenté devant la Commission «de la Cavitation», a une conclusion assez particulière. M. Rata déclare, et la Commission a été d'accord avec lui, que dans l'état actuel des choses la détection par le bruit permet de défìnir le début de la cavitation, mais ne permet pas d'en déterminer l'emplacement et l'amplitude.

M. Chevalier voudrait insister sur le point commun aux rapports de MM. RAta et Vercasson. Dans les deux cas, il s'agissait du collationnement d'informations existantes et on peut dire que ce collationnement a été fait de la manière la plus large possible en questionnant de façon précise les personnes directement au courant des sujets en étude.

Malgré cela, des conclusions plus nettes et surtout plus générales que celles qui ont été déposées par les rapporteurs sont actuellement impossibles à tirer. En particu- lier, que le bruit de cavitation permette de déceler avec précision le début d'apparition de la cavitation n'intéresse ni le constructeur ni l'exploitant.

La conclusion générale qu'il paraît important de tirer de ces deux rapports est donc évidemment qu'un gros travail de recherche reste à effectuer pour éclaircir en particulier ces deux points, et également qu'une grande prudence doit être de mise lorsqu'on parle de sujets de discussion aussi tentants que l'effet d'échelle dans les turbines Pelton ou le bruit de cavitation caractérisant le phénomène de cavitation.

M. Ie Président indique que M. Ghevalier est rapporteur de la Section "Machines》 et qu'il vient d'expliquer d'une façon très claire les conclusions auxquelles on arrive.

M. le Président s'étomne que personne n'ait de question à poser et remercie M. RATa.

\section{A review and critical examination of acoustic methods of observing cavitation}

\author{
BY A. RATA
}

\begin{abstract}
Cavitation seems to lend itself well to observation by acoustic methods. The aim in writing this report was to review the methods now in current use and, in particular, to ascertain their limitations.

A survey carried out at a number of different industrial research laboratories revealed-rather surprisingly - that they only very seldom used acoustic methods. It is a fact that a fairly large number of parameters have to be considered in determining noise level, and attempts to distinctly isolate their respective effects have so far failed to produce really satisfactory results.

Closer information on incipient cavitation and some indications on its development are obtainable from noise analysis, but if really significant measurement results are to be obtained, the transmitting properties of the cavitating medium should on no account vary during the tests
\end{abstract}


as, for instance, due to varying air content or gas dispersion effects. Acoustic methods are a useful auxiliary to visual observations in scale model work, and are in fact the only practicable method for industrial prototype tests. On the other hand, they cannot be claimed to provide a ready means of locating cavitation on a hydraulic machine component, nor is it yet possible to forecast erosion from the noise level of a given machine.

Cavitation noise can be investigated by the three following methods:-

(i) By measuring overall noise level;

(ii) By noise frequency spectrum analysis;

(iii) By noise amplitude spectrum analysis.

The overall noise level method (Figures 1 and 2) is both the one most commonly used and the most convenient. The pick-up, which is placed at a convenient point near the noise source in the machine, generates an EMF the values of which are measured over the entire frequency range covered by the pick-up. The effective value of the signal is usually the one considered, as it has the nature of an impulse.

In the frequency analysis method (Figures 3 and 4), a frequency spectrum is found for each considered point by measuring the energy content of adjacent frequency bands. These spectra distort depending on the degree of cavitation.

In the amplitude analysis method (Figures 5 and 6), on the other hand, the incident signal is divided up into bands of equal amplitude, and the frequency of occurrence of the signal peaks within these bands is measured. Each value of the cavitation parameter thus also gives an amplitude spectrum.

The results of these methods are analysed by plotting signal variation against cavitation parameter. The resulting curves show a frequently sharp rise in the signal level from a certain cavitation parameter onwards.

Both piezo-electric and electromagnetic pick-ups are used, with a frequency response range from several cycles up to several hundred kilocycles. They can be calibrated for pressure, though relative noise level measurements are frequently adequate for the purpose in view. These pick-ups are positioned as close to the noise source as possible, preferably at a wall, and are insulated from the machine by suitable flexible seals or joints.

Standard measuring instruments are used with the pick-ups, but should be of a very high input impedance type were combined with piezo-electric pick-ups. The use of thermocouple elements ensures that the signal is determined in terms of its effective value.

The three methods discussed have a number of additional aspects. Incipient cavitation is usually fairly well defined by the overall noise curve; the other methods do not throw any fresh light on the subject at the moment, but appear to indicate a future possibility of finding out more about cavitation development from spectral distortion.

Systematic experimental research will nevertheless be necessary to identify possible relationships between noise spectra, developing cavitation and the localisation of cavitation on difrerent machine components.

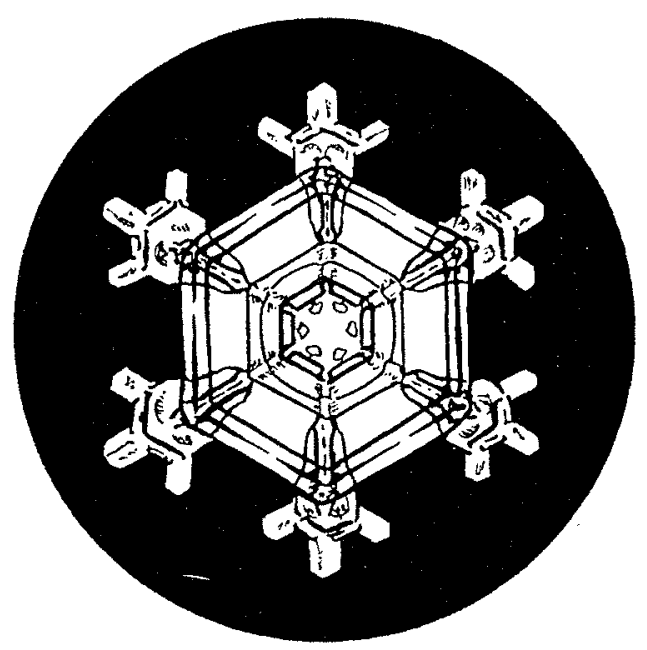

